\title{
User-Centred Multimodal Reminders for Assistive Living
}

\author{
Marilyn R. McGee-Lennon', Maria K. Wolters ${ }^{2}$, Stephen Brewster ${ }^{1}$ \\ ${ }^{1}$ Glasgow Interactive Systems Group, \\ School of Computing Science, \\ University of Glasgow, UK \\ (mcgeemr, stephen)@dcs.gla.ac.uk \\ ${ }^{2}$ School of Informatics, \\ University of Edinburgh \\ Edinburgh, UK \\ maria.wolters@ed.ac.uk
}

\begin{abstract}
While there has been a lot of research on the usability of reminders and alarms in the work context, the home has been somewhat neglected despite the importance of reminder systems for telecare and assistive living systems. We conducted a comprehensive mixed-methods study into the requirements for useable and acceptable reminders in the home. The study consisted of a questionnaire $(\mathrm{N}=379)$, 6 focus groups, and 7 home tour interviews. Our results highlight the need for highly flexible and contextualized multimodal and multi-device reminder solutions that build on existing successful strategies for remembering in and around the home. We suggest that developers of home care reminder systems should design for diversity, context, priorities, autonomy, shared spaces, and optimal care.
\end{abstract}

\section{Author Keywords}

Multimodal interfaces, personalisation, reminder systems, assisted living, telecare. focus groups, home tours, surveys

\section{ACM Classification Keywords}

H5.2. Information interfaces and presentation: User Interfaces (User Centred Design).

\section{General Terms}

Design, Human Factors.

\section{INTRODUCTION AND MOTIVATION}

People with disabilities or chronic long-term illnesses are increasingly being cared for at home in the community [5]. Home care and assistive living solutions are crucial for enabling this user group to stay active and independent for longer in their own homes [6]. Reminder systems can be an important part of the assisted living telecare package. Many service users have problems remembering information, tasks, and chores in and around the home. This can be a consequence of cognitive ageing, the conditions for which they need care, or the medications they need to take.

Permission to make digital or hard copies of all or part of this work for personal or classroom use is granted without fee provided that copies are not made or distributed for profit or commercial advantage and that copies bear this notice and the full citation on the first page. To copy otherwise, or republish, to post on servers or to redistribute to lists, requires prior specific permission and/or a fee.

CHI 2011, May 7-12, 2011, Vancouver, BC, Canada.

Copyright 2011 ACM 978-1-4503-0267-8/11/05...\$10.00.
Forgotten tasks may be as trivial as returning library books or as crucial as taking important medication. Being able to remember these tasks can be the difference between having to send people to care homes and enabling them to age in place, in the comfort of their own home.

Even though many people with care needs would benefit from assisted living technology, uptake is still comparatively low $[3,9]$. In order to increase adoption and ensure successful implementation of assistive living technology, we need to provide users with effective, accessible solutions that are enjoyable to use. These solutions need to be adaptable to a set of devices and interaction modalities that will depend on user needs, abilities and preferences as well as on available services and budgets.

The home is a special interaction space. Even though it is private and intimate, it is often shared with other people $[11,15]$. A home is a very personal space for everybody who lives there, not just for the intended user of the system but also for a spouse, children or even visitors. This means that we need to design reminders that are both useable and acceptable to a range of direct end users, i.e. the intended recipients of reminders, and indirect users, i.e. people who can perceive the reminder message despite not being the intended recipients. Reminders need to be adapted to multiple, sometimes conflicting, stakeholder needs [17], they need to be appropriate in a variety of changing contexts and they need to accommodate a wide range of ability levels [22].

Existing approaches to reminder design only partly address these complex issues. There is a clear need for guidelines that allow designers and developers (and where appropriate end users) to create configurable and multimodal reminder systems which can rise to the challenges of home care. The systems that are discussed in the literature on alerts in the work place $[26,28]$, telephone-based health care reminders [2], and general reminders in the home [16] are often unimodal, and personalisation is limited to one or two parameters, such as preferred times for reminder calls. On the other hand, studies that take a wider view of design issues, such as $[4,19,20]$, tend to focus more on home monitoring of frail older people than on reminding. 
With the present study, we address this gap in the literature by studying reminder design using a rich mixed-methods approach in order to understand both trends and deeper social context. Themes that emerged from a survey and focus groups were cross-validated in the home. We worked with a broad range of users, including older people and people with sensory impairments. We distil our findings into six design guidelines that can be used to improve the design of future homecare systems.

\section{DESIGNING TECHNOLOGY FOR THE HOME}

Home care and assisted living pose very specific challenges for design. The key issues can be clustered around three main themes; complexity of care needs, the multi-user and multi-stakeholder nature of home care, and the need for well-defined pathways and accountability [17].

\section{Complex and Dynamic Care Needs}

Interventions for people who are cared for in the community are complex [13]. Many people require care for two or more different conditions, which may or may not be causally related. Reminder systems must be able to accommodate such holistic care plans and the team of formal and informal carers that manages them [4, 19]. Due to the potential deluge of reminders for such complex interventions, it is important to choose delivery options carefully, otherwise vital messages may be missed and the system will not be effective.

We advocate a person-centred approach to care similar to [4] where needs and preferences are regularly reviewed and stored in a format such that they can be easily communicated to relevant stakeholders including end users. To accommodate this approach, reminder systems need to be highly modular, flexible and easy to configure.

Care plans change over time not just because of new care needs but also due to changes in the available devices and services. Care provision may need to shift from people to technology because the budget for formal carers is cut or informal carers are no longer able to provide the level of care they used to give. People's beliefs and behaviours can also change. Users who were highly sceptical of technology may become advocates after having experienced the difference a well-designed system can make and vice versa. Good reminder systems need to be able to accommodate these changes.

Making reminders multimodal is particularly important given that the acuity of all sensory modalities declines with age [1], often as a consequence of the condition that caused the original care needs. For example, diabetes may lead to impaired vision and, due to peripheral neuropathy [7], to an impaired sense of touch. The number of people with both significant vision and hearing impairment is set to increase dramatically in the future, partly as a consequence of the ageing population [25]. Alerts that are based on touch and smell are particularly important for these users.
While it is tempting to use only those modalities for reminders where acuity is relatively well-preserved, it is in fact important to make reminders in all modalities as accessible as possible to people with varying abilities. For example, even though touch can function as an alert to take medication, users might not remember which medication they need to take or how many of each pill are required. This information might need to be presented graphically or sonically. Designers need to work with care professionals to devise a strategy for accommodating different levels of sensory impairment. An example for such a strategy for tasks that require dexterity can be found in [27].

\section{Home Care is Multi-User and Multi-Stakeholder}

In the home care reminder context, we define users as people who choose (or in some cases are selected) to receive reminders. Direct end users can include the person with care needs, family, friends and formal carers. For example, a system might remind all members of the household of an appointment, or it might remind the formal carer of tasks such as checking the medicine cabinet.

Stakeholders are defined as those who can directly or indirectly specify requirements and add, delete or change reminders or the information the system produces. Examples include the health and social care team that manages care, the providers of specialist housing, telecare equipment providers, informal carers such as friends and neighbours, and people who live in the same home but are not directly involved in care.

Many potential conflicts regarding reminder delivery can arise between users and other stakeholders. For example, a user may prefer auditory reminders, but due to a hearing impairment, these reminders need to be so loud that the neighbours can hear. A formal carer may have decided to use a loud beep to remind a user to lock the doors at night because this has been identified as a safety threat, but the user perceives this sound to be highly disruptive and disturbing. These conflicts need to be resolved so that the reminders remain effective while still being acceptable to the user and those sharing the home interaction space.

\section{Pathways and Accountability}

Since reminder systems are multi-stakeholder, it is particularly important to identify clear pathways for resolving any conflicts. These pathways need to include explicit mechanisms for seeking user input and validating the selected options. The different goals and needs that underpin each conflict need to be identified [8]. In resolving conflicts, clinical efficacy needs to be balanced with an acceptable user experience and institutional requirements.

Ideally, the care team should have a variety of devices, modalities and strategies at their disposal. For example, potentially disruptive auditory reminders could be delivered straight to the user's hearing aid. Disruptive beeps might be replaced by a light next to the user's bed that changes colour when the doors are locked. Finally, it needs to be clear who owns the data generated by the reminder system 
and who controls the system's behaviour (e.g. who is responsible for configuring the system or repairing faulty devices)

\section{AIMS AND APPROACH}

In order to inform the design of configurable, adaptable reminder systems for the home, we sought to determine

- What users need to be reminded of, i.e. the tasks they forget to do in and around the home;

- Why users need reminders, i.e. reasons for forgetting;

- What strategies are used to remember, i.e. what techniques and technologies people currently use;

- How users would like to receive reminders, i.e. the devices available and preferred by users and the modalities available and accessible to users.

Our user requirements work consisted of a full year of user engagement activities with various user panels (older adult volunteers), social work professionals, charities and groups specialising in sensory impairment, and technologists and technicians working in the area of assistive technologies. We designed a mixed methods approach including a questionnaire (to gain a wide range of views and trends), focus groups (with specific groups of older adults and adults with sensory impairments) to more fully understand trends and responses in the questionnaire data and home tour interviews (with older users in their home) to probe how social and contextual issues of living can influence the usability and acceptability of reminders in situ.

Using any one of these techniques on its own is common in HCI but each has its own well-reported problems. Surveys can be cheap to administer but often have a poor return rate. It is difficult to encourage people to answer anything but forced choice questions, and it is hard to get respondents to explain their choices. Other techniques are often required to understand quantitative response patterns.

Focus groups are a good way for HCI researchers to probe issues in more depth and to obtain feedback on prototypes. In discussion, participants will share and elaborate on stories and insights that do not fit into the boxes of a questionnaire form. It is also much easier for users to comment on reminder technology if they can see, hear and touch, and smell examples. However, what people claim they do may be different from what they actually do. An ethnographic component is needed to address this issue.

Cultural probes and in depth interviews in the home have been shown as successful ways to engage with older users [11]. In this study, we used home tours to gather a rich contextualized understanding of how people use reminders in their own homes and the social and physical context in which remembering succeeds or fails. Successful reminder strategies could be demonstrated in practice and important details could be observed and recorded (photos and audio) that might not be reported during a focus group.
The temporal sequence of the three methods was designed to support our overall research strategy. The main online data collection for the survey finished before the focus groups began, so that these results could inform the focus groups. Likewise, the home tours started after a few focus groups had already taken place. Therefore, the researcher who conducted the home tours was already highly sensitized to different reminder strategies, which made it easier to observe them in context.

Using these three techniques in combination provided a much richer understanding than any one of the techniques alone. While the survey enabled us to establish the range of preferences for devices and modalities that should be accommodated in reminder system design, the focus groups allowed us to discuss the pros and cons of different strategies in depth, and the home tours gave a vivid picture of reminder strategies people really use in their homes.

\section{STUDY DESIGN}

We included younger, middle-aged and older users with and without sensory impairments in our study because people of all ages can be stakeholders in home care systems. For example, some of our younger participants had sensory impairments or cared for people with sensory impairments. We were also interested in generational differences in reminder preferences. Since today's middleaged users will be tomorrow's older generation, reminder solutions that would be difficult to deploy widely today might be almost universally acceptable in a decade's time.

\section{Questionnaire}

Since we wanted to explore the diversity of the design space, we chose a broad sampling strategy. Online and postal versions of our questionnaire were distributed through email, social networking sites, Web sites, local user panels, senior's forums, charities and community health partnerships across Scotland. Our sample was deliberately skewed towards older people, as they are more likely to require home care. More information about the survey can be found in [29].

The questionnaire was designed based on previously validated topics and question formats as well as in-depth qualitative work with older users on assistive technology and home care projects by the research team $[3,15,16]$. We used a balance of open ended and multiple choice questions and piloted the questionnaire with older people from our user panel. The online survey can be found at http://www.multimemohome.com/get_involved. To ensure legibility, the postal version was typeset with a large font.

We present an analysis of all 379 questionnaires (206 online, 173 postal) that had been returned by the end of July 2010. The return rate for the postal questionnaire was $19 \%$. $13 \%$ of respondents were aged between 18 and 30 (younger group), $46 \%$ between 31 and 60 (middle-aged) and 41\% were aged 61 and above (older). The ratio of female to male respondents was 2:1. 34\% lived alone (older group: 51\%), $2 \%$ with a flat mate or lodger, and the remainder lived with 
a partner or family (older group: $48 \%$ ). Only $3 \%$ lived in sheltered accommodation.

Compared to the general UK population [21], our sample is highly computer literate. While only $24 \%$ of UK citizens aged 60 and above use the Internet regularly, $75 \%$ of our older sample were online (postal: 55\%). Nevertheless, our older sample is just as likely to use a mobile phone regularly as the average older person (45\% in our survey versus $48 \%$ UK average).

$20 \%$ of respondents reported problems with their vision, $17 \%$ had hearing difficulties and $4 \%$ each had a reduced sense of touch or smell. $27 \%$ of our sample said they had problems with their memory (younger: $6 \%$, middle-aged: $13 \%$, older: $48 \%$ ). $30 \%$ of our sample reported chronic care conditions (older: $50 \%$, middle-aged: $18 \%$, younger: $4 \%$ ). Of the people with a care condition $24 \%$ had heart conditions including angina, $18 \%$ had mobility problems, often due to arthritis and $17 \%$ had hypertension. Other conditions included diabetes (14\%), asthma/breathing problems $(10 \%)$, fatigue $(9 \%)$ and cancer $(6 \%)$.

\section{Focus Groups}

We conducted 6 focus groups (summarized in Table 1) with older adults, assistive technology technicians and younger adults with sensory impairments over a period of three months, starting after the online questionnaire survey had gone live. This was feasible because the survey was already based on extensive previous qualitative work.

\begin{tabular}{|l|l|l|l|l|}
\hline$\#$ & $\mathbf{N}$ & Location & User Group & Impairments \\
\hline 1 & 6 & Glasgow & older users & $\begin{array}{l}\text { Hearing (slight, } \\
\text { one; severe, two) }\end{array}$ \\
\hline 2 & 5 & Glasgow & $\begin{array}{l}\text { younger } \\
\text { users }\end{array}$ & Vision (one) \\
\hline 3 & 2 & Edinburgh & older users & $\begin{array}{l}\text { Memory (slight, } \\
\text { one) }\end{array}$ \\
\hline 4 & 2 & Falkirk & technicians & $\begin{array}{l}\text { Hearing (severe, } \\
\text { one) }\end{array}$ \\
\hline 5 & 2 & Falkirk & older users & Hearing (both) \\
\hline 6 & 2 & Glasgow & $\begin{array}{l}\text { younger } \\
\text { users }\end{array}$ & Deaf (both) \\
\hline
\end{tabular}

Table 1: List of focus groups conducted.

The focus groups were structured around the main research questions outlined above. Reminder devices and modalities were illustrated using technology probes for four of the five modalities covered in the survey - speech, non-speech audio, touch, and smell. Participants were also asked to respond to each of the research questions on 'sticky notes' and organise the resulting themes into hierarchies of importance to them. This was used to stimulate further discussion. The same researcher acted as facilitator across each focus group. An additional researcher took notes, observed and demonstrated speech technology probes.

\section{Home Tours}

We conducted seven Home Tours (summarized in Table 2) with users in their own homes. Each tour consisted of an interview and a tour of the home to capture current reminder strategies. Interview responses and the home owner's verbal comments during the home tour were recorded using a digital audio recorder and later transcribed for analysis. Photographs were taken of objects/items that were used to remember things and strategies that were used to remind people to do things. The photos were stored digitally and tagged in relation to the context of the interview and observations. More detailed information about the Home Tours can be found in [18].

\begin{tabular}{|l|l|l|}
\hline$\#$ & Participants & Description \\
\hline 1 & Mrs. M & Single older woman (76) \\
\hline 2 & Mrs. H & Single older woman (74) \\
\hline 3 & Mr. PL & Single man (39) \\
\hline 4 & Mrs. R & $\begin{array}{l}\text { Single older woman (89), } \\
\text { Parkinson's, mobility, received } \\
\text { home help }\end{array}$ \\
\hline 5 & Mr. \& Mrs. G & Husband (65) and wife (64), \\
\hline 6 & Mr. \& Mrs. L & Husband (72) and wife (72) \\
\hline 7 & Family PC & $\begin{array}{l}\text { Grandmother (65), mother (37), } \\
\text { two children (11,9) }\end{array}$ \\
\hline
\end{tabular}

Table 2: List of home tours conducted

\section{METHOD}

\section{Statistical Analysis}

Unless otherwise specified, chi-square tests were used for analysis of multiple-choice items from the questionnaire. For correlations, Spearman's correlation coefficient was used. P-values were reported at three significance levels, $\mathrm{p}<0.01\left(^{*}\right), \mathrm{p}<0.001\left(^{* *}\right)$, and $\mathrm{p}<0.0001$ or lower $(* * *)$.

\section{Qualitative Analysis}

Qualitative data was stored and managed using the data management software NVivo. The salient responses and themes for each of the research questions were identified and categorized using the Framework Approach [24].

\section{RESULTS}

\section{What Do People Forget and Why?}

$41 \%$ of older respondents in the questionnaire said that they forgot to do certain things in and around the home often or all the time, as opposed to $32 \%$ of middle-aged and $17 \%$ of younger respondents. However, when specifying what tasks they forget, older people ticked fewer boxes than younger or middle-aged people. In particular, they were less likely to report that they forget daily chores (older: $4 \%$, middleaged: $14 \%$, younger: $22 \%)$ and paying bills $(10 \% ; 32 \%$; $42 \%$ ). All age groups were equally likely to forget medications and appointments. 
The most popular responses across the focus groups for the things forgotten around the home fall into five groups: chores around the house (closing windows and doors, putting out bins, unloading the washing machine), preparing to go out (remembering to take keys, umbrella, or hat), appointments, health care-related tasks (taking tablets), and ambient or place-specific reminders (what I went into a room for, where I have put something, or when to get off the bus).

The things that cause people to forget tasks were similar across all methods used. Memory problems, failing to use reminder strategies, and distractions, e.g. by phone calls or more important events, were all mentioned. People also stated that they tended to forget tasks that were not important or urgent. The only significant difference between age groups in the survey was that middle-aged people were more likely to say they were too busy $(67 \%)$ than younger $(48 \%)$ or older $(34 \%)$ people $(\mathrm{p}<0.0001)$.

Stress, tiredness, and low mood were cited as further reasons for forgetting. Some respondents mentioned that physical problems prevented them from remembering or completing tasks. These problems were not just caused by ill health, but also by the medications people were taking.

\section{How Do People Remember?}

The highly varied strategies people claim to use can be grouped into five categories: paper-based (e.g., calendars), technological and specialized (e.g., mobile phone reminders and pill boxes), temporal (e.g., integration into routines), people-based (e.g. phone calls from friends) and physical (e.g., placing books to be returned near front door).

In the multiple-choice items of the survey we mainly explored paper-based and technology-based reminders. Figure 1 shows how often our respondents use six representative strategies. The other three reminder types emerged from the open questionnaire items, the focus groups and the home tours.

\section{Paper-based methods}

Calendars and diaries were the most popular, followed by to-do lists and sticky notes. In the questionnaire sample, older people were more likely to use diaries and calendars, and were less likely to set alarms or use an electronic personal organizer (see Figure 1).

While the survey revealed the central role of paper-based reminders, the home tours allowed us to fully document the complexity of such systems. Many people had multiple calendars, diaries and notebooks and complex systems for annotating them and working between them (see Figure 2(a)). This could involve one calendar for the family events and appointments and one for an individual's activities. It also included strategies for copying selected diary appointments on to the calendar while some remained in the diary only.

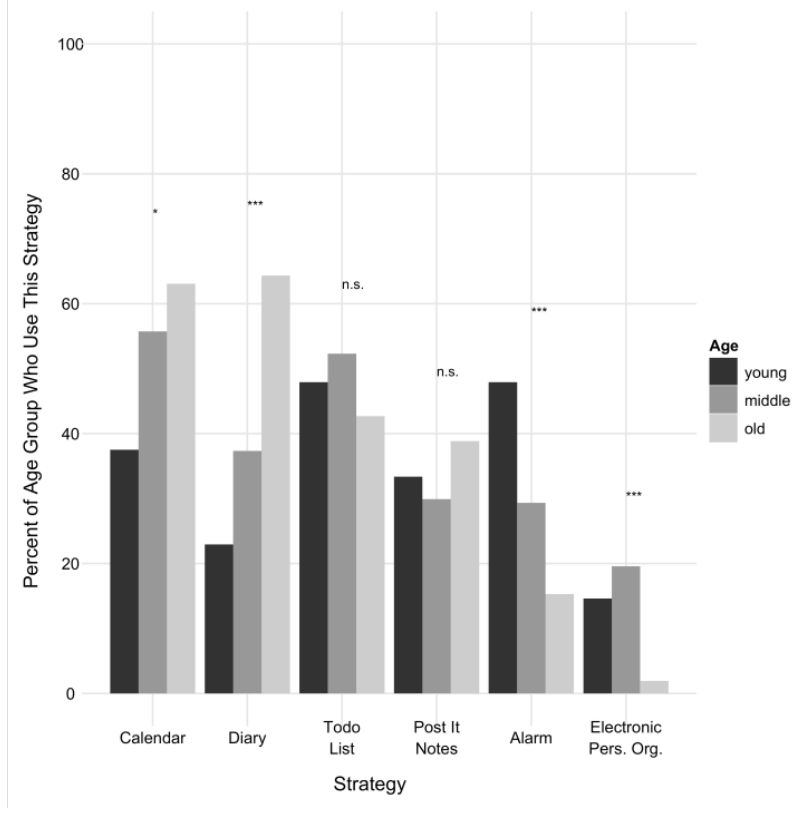

Figure 1: Reminder methods used by respondents

Diaries and calendars were used as memory aids for information about past events needed to plan future ectivities. One couple noted down when they had last replaced watch batteries so they knew when the next replacement was due, and one person recorded information gained at important medical appointments to help him manage his care.

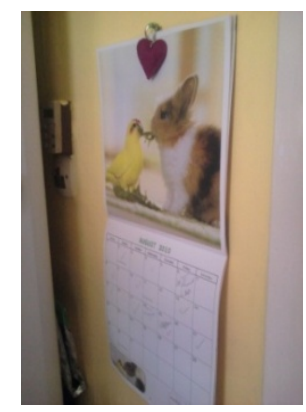

(a)

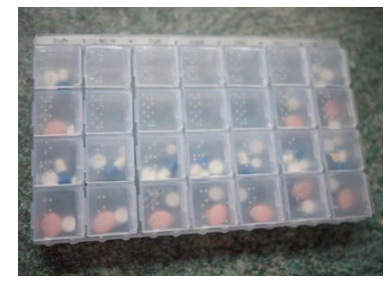

(b)
Figure 2: Paper based (calendar) and specialized reminders (pill box).

\section{Technological/specialised reminders}

Technological and specialised reminders mentioned by our participants included reminders sent to themselves on their mobile phones, computer applications, timers worn on the person and specialized solutions such as pill boxes to remember to take their medication (Figure 2 (b)).

\section{People-based reminders}

Several respondents mentioned people-based strategies, such as relying on friends and family to remind them of important tasks. Several people in the survey also described serving as the main personal reminder system for a family member with dementia. 


\section{Temporal reminders}

Temporal reminder strategies emerged as central throughout our research. A common strategy was to integrate tasks that would otherwise be forgotten into routines, e.g. "I never leave the bathroom without looking at taps" (male, 46-60, survey). This plays to older people's strengths, because this age group found it particularly easy to remember daily chores. Temporal cues could be external, such as radio programs that served as indicators of the time.

\section{Physical reminders}

The importance of a close fit between reminder strategy and daily life is illustrated by the large variety of physical reminders, which were a prominent feature of our home tours. Many people left visually meaningful or salient objects such as packaging in places where they would noticed. For example, people left out empty food packaging to remind them to $\log$ their daily food intake on their computer. Other examples include leaving library books by the door as a prompt to return them (see Figure 3(a)). Technological reminder strategies such as pill boxes were supplemented by the physical reminder of empty medication packaging near the television.

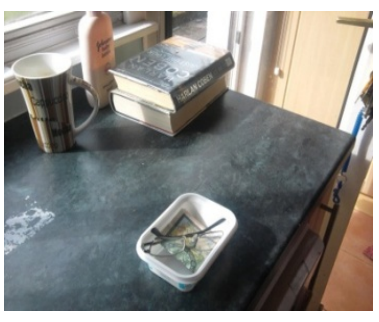

(a)

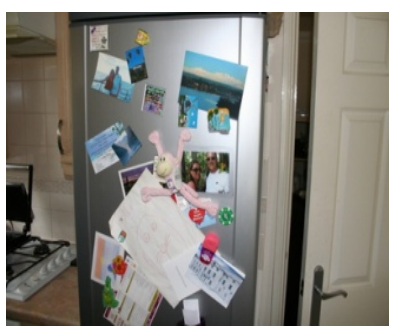

(b)
Figure 3: Physical/visual reminders

Kitchen surfaces such as a fridge or a blackboard/whiteboard (Figure 3(b)) often acted as a todo list. The surfaces were used for leaving messages to other family members, detailing shopping lists and posting reminders of upcoming appointments and things to remember to take out of the house.

Physical context and spatial location even affected the reminder system used. A questionnaire respondent listed, for example, "[a] shopping list on the freezer door. A to-do list on my mobile phone with alarms attached to the individual items [...] "' (female, 31-45, survey).

\section{How Would People Like To Receive Reminders?}

In the questionnaire, we collected extensive quantitative data on device and modality preferences. First, we asked respondents whether they would prefer to receive visual, tactile, olfactory, speech, or non-speech audio reminders. $21 \%$ of questionnaire respondents did not state any preference, $46 \%$ expressed a preference for one modality, $21 \%$ named two modalities and $12 \%$ found three or more modalities acceptable. The only significant difference between age groups was that older people were less likely to favour visual reminders $(38 \%$ positive versus $60 \%$ for middle-aged and $69 \%$ for younger). Interestingly the selfreported impairment of a particular sensory modality did not affect whether the user would like to receive reminders using that modality.

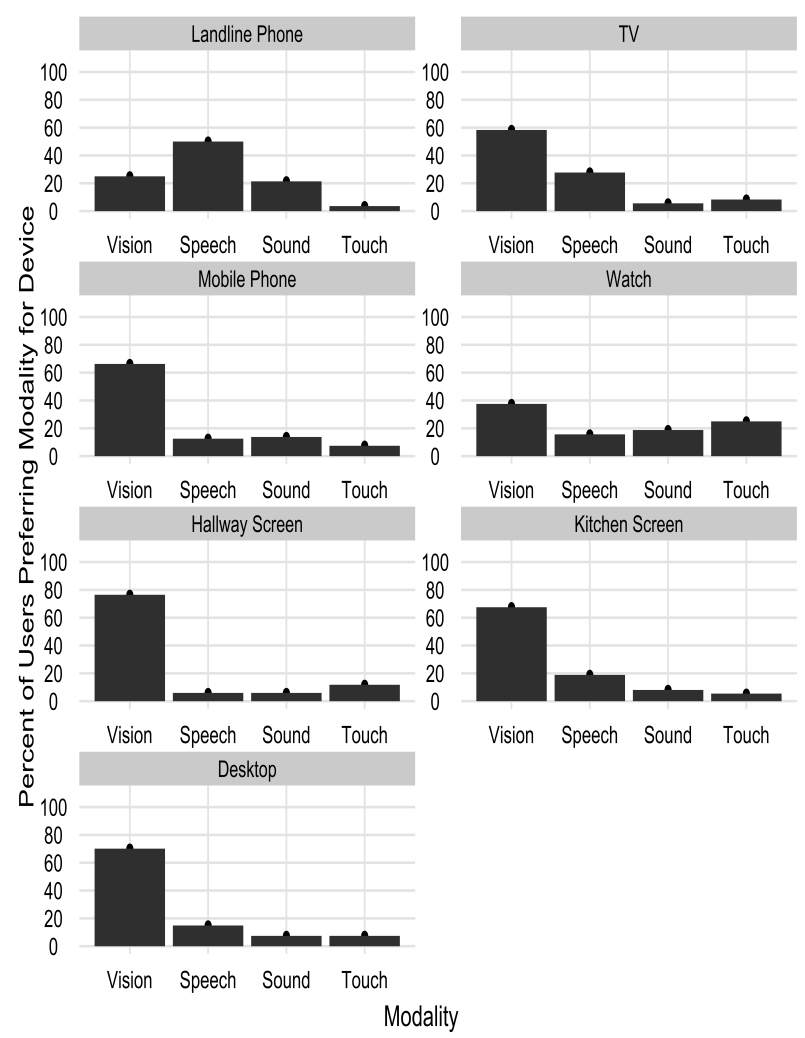

Figure 4: Modality preferences by device for people who prefer only one modality.

$66 \%$ of questionnaire respondents who expressed a modality preference wanted visual reminders, 37\% liked speech, 30\% non-speech audio, 23\% touch and 3\% smell. Users who liked speech were unlikely to choose touch (Spearman's $\rho=-0.41, \mathrm{p}<0.000$ ), and users who preferred vision were unlikely to favour non-speech audio $(\rho=-0.24$, $\mathrm{p}<0.01)$. There were also weak dissociations between speech and non-speech audio $(\rho=-0.19, \mathrm{p}<0.05)$ and vision and touch $(\rho=-0.18, p<0.05)$.

Device preferences were even more varied than modality preferences. $83 \%$ of respondents expressed a preference for one or more devices. The most popular device was the mobile phone (55\% of those who specified a device), followed by the desktop computers $(43 \%)$, a screen in the kitchen $(30 \%)$, the watch $(29 \%)$, the TV $(25 \%)$, the landline phone $(20 \%)$ and a screen in the hallway $(15 \%)$.

The main difference in device-related preferences between age groups concerned phones. $40 \%$ of our older sample wanted reminders to be delivered by landline phones, whereas fewer than $5 \%$ of younger and $8 \%$ middle-aged users favoured this mode of delivery $(p<0.001)$. Conversely, $83 \%$ of younger and $67 \%$ of middle-aged 
adults, but only $32 \%$ of older adults, chose mobile phones $(p<0.001)$ as a reminder delivery method. Only a third of those older people who liked reminders on landlines also wanted to receive them on mobile phones.

To investigate associations between devices and modalities, we looked at the device preferences of 174 respondents who selected only one modality for reminders (see Figure 4). For each device, we determined how many of the people who had chosen this device wanted to receive visual, haptic, spoken, or other auditory reminders. Smell was excluded because there were too few data points. The results are summarized in Figure 4. Visual reminders dominate for screens and desktops. Of the two mobile devices, mobile phone and watch, the watch shows the greatest range of desired modalities. Mobile phones, on the other hand, appear to be mainly associated with visual reminders such as text messages. Speech and sound figure prominently in reminders delivered via TV and landline.

In the qualitative component of our work, five strong themes emerged regarding the type of reminders people would find useful and acceptable: adaptability, integration, privacy, accessibility, and choice.

\section{Adaptability to User}

Users wanted a system that can be adapted to their own preferences and capabilities. "I mean different people want different things don't they... they would need to be able to choose what suited them I suppose..." (FG1, male, 71-85). Several survey respondents highlighted using paper calendars and diaries instead of technology, extending strategies people already successfully use. Some also wanted to receive reminders by post, when appropriate. Speech output in particular should be configurable. Individual reactions to gender, politeness, accent and length of message were very strong and varied. "I mean I would want a short abrupt kind of message if it was an urgent matter but if it was like 'take the dog out' then the longer softer ladies voice would be nice." (FG5, female, 71-85).

The family home tour revealed clear generational effects. The middle-aged mother discussed solutions that could exploit her mobile phone, the young children were very positive towards wearable devices and the grandmother was keen to exploit mainstream technologies such as their TVs. The youngest participant summarized the need for personalisation as follows. "I would want it to like a hair band or a watch ... you know telling me to remember my school stuff ... but then mum could get her reminder to her phone cos she is always on that..." (Tour 7, Male, 9).

\section{Seamless Integration into Life and Home}

Users wanted a reminder system to be easily integrated into their daily life and their physical surroundings. "Maybe like a digital photo frame or something ... but it would have to look okay wouldn't it - you wouldn't want some big box in your living room..." (FG2, male, 46-60). Successful reminder systems need to strike a delicate balance between being prominent enough to attract users' attention, but subtle enough not to be perceived as intrusive or distracting. Audio reminders, in particular, can startle or scare users if played unexpectedly.

Screens should be placed where users are most likely to see them, such as in the living room or kitchen. Many people favoured the idea of a controllable panel in the hallway. Users also liked the idea of more ambient display technologies (such as a coloured light) that act as generic reminders. "Sometimes I just need reminding that I have to remember something you know ... so I wouldn't even need to hear 'take your pill' I would just know if I heard the bleep that I have to take my pill." (FG3, female, 85+).

Surprisingly, location was also an important factor for phone-based reminder systems. People were concerned that they might not be able to get to a landline phone in time or that they might not be able to hear mobile phone reminders because they did not know where their mobile was. A final aspect relevant to the theme of seamless integration was robustness. Quite a few users doubted the reliability of technology, and they were wary of the disruption that a malfunctioning reminder system was likely to cause.

\section{Privacy in Shared Spaces}

Conflicts due to privacy and shared interaction spaces were highlighted in particular during the home tours - they did not emerge as strongly in the focus group and survey components. "I wouldn't want all my messages sent to the system so that everyone could see them. I would need to be able to pick which ones went to my phone [mobile] so that only I could see them." (Tour 7, Female, 37)

\section{Accessibility for People with Impairments}

Many users raised concerns about usability issues such as the font size of visual reminders and intelligibility of audio reminders. However, this does not mean that people with a visual or auditory impairment would not use visual or auditory reminders, In our focus groups with people with severe hearing impaired people (including Deafness), one person said "Some speech is okay with my hearing aid ... I mean it would depend on the volume and quality [of the speech] wouldn't it..." (FG6, male, 18-30, Deaf). Thus, even for people with severe sensory impairment, the choice of delivery modality is not straightforward.

\section{Choice between Diverse Modalities}

Overall, modality preferences depended did not just depend on the modality itself, but also on the amount of information to be conveyed with beeps and vibration favoured for more simple alerts. Some users wanted to be able to remove reminders once the associated task had been completed while others would like a view of the history of reminders they have attended to.

Participants reacted positively to the idea of smell and tactile reminders but this may have been due to the novelty of these delivery methods. "Yes...I mean like a horrible smell to tell me the washer [washing machine] needs emptied - that would be ideal...". (2G5, female, visually impaired, 31-45). This illustrates the effect of technology 
probes - in the survey, respondents were far less likely to comment on olfactory reminders.

\section{Why do Reminders Fail?}

Reminder strategies are only effective if people can adhere to them. Adherence can be affected by health problems. For example, a person with depression may choose not to act on reminders when feeling low. A few users argued that their needs were so complex that they could not be met by a reminder system alone. For example, one older male respondent was a diabetic who sometimes forgot his insulin. Since double doses of insulin can result in complications, what this person needed was not simply a reminder to take his insulin, but also a way of monitoring that he had already taken it.

The very routines that are crucial for establishing successful temporal reminder strategies can also interfere with adherence. Participants noted that existing habits can interfere with new habits that need to be established as a response to care needs. Conversely, a lack of routines means that users lacked a temporal scaffold to which tasks and events could be attached.

Some of the respondents who did not state any modality or device preferences disliked the idea of automated reminders because this would result in an external locus of control, i.e. others having power over their lives. Others did not think they needed them, reflected in comments like "don't feel I am in this category" (survey, male, 85+). For these users, reminder systems were a threat to their identity and autonomy.

\section{OUTPUTS AND IMPLICATIONS FOR DESIGN}

Our methodology allowed us to develop a rich picture of the breadth and complexity of the design space involved in creating reminder solutions for people from a range of age groups and with complex care conditions. We found that if novel reminder systems are to be usable and acceptable they will need to exploit existing metaphors and strategies of reminding. Knowledge of the home and the structure of daily life is also required to ensure that that reminder systems can be seamlessly integrated into users' lives.

In this section, we synthesize our findings into a set of six design guidelines. These are intended to be used by health and social care professionals to inform their prescription and deployment of assistive technologies involving reminder systems, and by designers and technologists to produce systems that are more likely to be used and accepted in the homes of users in the future.

\section{Design Guidelines}

\section{Designing for Diversity}

Reminders need to be highly personalizable, because one size does not fit all. Phone-based solutions are a good example: Even though mobile phones are the basis of many telecare packages, less than half of the older people we surveyed used their mobiles regularly; instead, $40 \%$ of this age group wanted to receive (mostly spoken) reminders on their landline phone. This limits the extent to which mobilephone based location-sensitive solutions like PlaceMail [12] can be deployed with the current generation of older people.

Personalisation is particularly important if users are to successfully integrate the reminders into their identity as a person with a chronic condition. For example, in their study of the mobile health management system MAHI, Mamykina et al. [13] found that each participant used MAHI in a unique way that was consistent with the way in which they approached their condition and its effect on their life. This was only possible because MAHI was sufficiently flexible. We would expect to see a similar picture for any deployed reminder system.

The associations we found between age groups and devices or modalities are tendencies, not rules. We would strongly caution against creating reminder technology packages that are mainly differentiated by the intended age group. Each person weighs the tradeoffs between modalities differently, and therefore, should be able to choose from a range of options. Age might however influence the interaction techniques that users are familiar and comfortable with and therefore find most acceptable. Since our older user group consists of people over the age of 60 , their preferences provide a good estimate of the design space for accommodating the oldest old of the next decade. Technologies that are required to cater for today's older users may be all but obsolete by the time our middle-aged group might require assistive living solutions a couple of decades from now.

Many people with sensory impairments wished to receive reminders in the impaired modality. Their wishes should be accommodated as much as feasible, because reminders delivered using unwanted modalities are more likely to be ignored or switched off. Designers therefore have an obligation to provide visual, auditory, and haptic reminders that are accessible and usable even for people with mild to moderate impairments of these modalities. People who configure reminder systems (such as health or social care workers) also need to be aware of this so that modalities are not excluded from a tailor-made solution purely because of a known impairment.

In order to support multimodal multi-device reminder delivery, systems should monitor what devices are currently available and people who configure systems should monitor what the users' current needs and preferences are. If there are any significant changes, systems should be adaptable with a minimum of effort. An example of such a highly configurable system is presented in [14].

\section{Designing for Context}

Contextualization figures heavily in the successful reminder strategies that emerged from the data. Reminders are most effective when delivered at the right location, at the right time and through the right device. Systems should be configurable based on the devices that are acceptable and available to the user. There are strong synergies between 
location and device. For example, many people favored reminder screens at the hub of the home, which is often the kitchen [11] or the living room. These can be used to remind inhabitants of general tasks and schedules, while a screen by the front door can be used to alert people to security precautions when leaving the house, for example.

Temporal contextualization is also a powerful reminder strategy. Our results suggest that integrating tasks and reminders with a person's habits and routines is key to effective, personalized reminder solutions. Such integration requires sophisticated planning algorithms that combine knowledge of the user's schedule and habits with information about the nature and duration of the tasks that need to be supported [23]. This is also the point where data from monitoring devices like bed sensors can be integrated.

\section{Designing for Priorities}

Reminders should reflect the urgency of the task. This helps users decide whether and when to attend to the reminder. It also prevents overload, which may result in users ignoring reminders on principle. Users should be able to choose appropriate modalities depending on urgency. For example, a loud spoken reminder could warn the user that the stove needs to be switched off urgently, while ambient lights might be as a gentle reminder of an upcoming appointment.

\section{Designing for Autonomy}

Autonomy is a fundamental issue in the design of pervasive technology for healthy ageing [10]. Reminder systems should support users as autonomous agents who are in control of the system, not the other way around. For example, users should be able to silence reminders that are no longer required. Another important option are simple 'reminders to remember'. These more ambient reminders are similar to reminders that people without care needs use, which makes them potentially less stigmatizing and, crucially, support users' ability to remember tasks themselves.

\section{Designing for Shared Interaction Spaces}

As soon as other people share the home with the intended recipient of the home care reminders, issues of privacy and obtrusiveness arise. Reminders that cannot be easily interpreted by visitors provide privacy, especially if users do not want to be seen as needing care. For example, while spoken reminders are explicit, clear indicators of care needs, the meaning of non-speech sounds such as Earcons is opaque unless explained [16]. At the same time, reminders should not unduly disrupt other people living in the home. While reminders that can only be perceived by the recipient, like haptic messages, do not disrupt other members of the household, the complexity of information that can be transmitted may be too limited to be useful. It is particularly important to consult with all relevant stakeholders to ensure the right balance is struck.

\section{Designing for Optimal Care}

The guidelines detailed above need to be weighed against the imperative of delivering good care. While it is important for adoption that reminders are acceptable to the end user, guidelines may need to be violated for important tasks, such as taking vital medication.

\section{Limitations}

With this study, we aimed to scope out a design space that would reflect the needs and preferences of people without major cognitive impairments. We made this decision because (i) many chronic conditions that require home care involve at most mild cognitive impairment and (ii) telecare solutions for older people may be implemented before they are needed to ensure the systems are in place and familiar to users when they are needed. Detailed specific guidelines for adapting interfaces to people progressing through dementia can be found in [19].

It is also worth noting that our sample consisted of native speakers of English. Therefore, appropriate care should be taken when extending them to different ethnic or cultural groups.

\section{CONCLUSIONS}

The guidelines presented have emerged from a large study where a common set of research questions was investigated using three highly complementary user requirements methods; a survey, focus groups and home tours. Emergent themes were cross-validated and triangulated across all three sources of data. Including a large postal element to our survey allowed us to reach a wide range of older users with and without care needs and sensory impairments. The focus groups allowed us to target specific user groups more precisely and to obtain feedback on somewhat unusual reminder strategies using technology probes. Finally, home tours allowed us to explore reminder strategies and needs in the homes of users and to gain a richer understanding of the social and physical context that might influence what is and is not acceptable in electronic reminder systems.

The next step is to test our design guidelines in practice. To this end, we plan to evaluate different multimodal reminder strategies under controlled laboratory conditions, before implementing our findings and guidelines in a pilot reminder system that will be evaluated in longitudinal home trials with users. Throughout this empirical validation phase, we will be working with system configuration experts, health care professionals, and end users to achieve the highly flexible and adaptable assistive living reminder solutions that users both want and need.

Given our findings, we have argued that reminder systems for assistive living need to be designed for diversity, context, priorities, autonomy, shared interaction spaces, and optimal care. In order to achieve these goals, reminder systems need to be configurable to accommodate a range of needs, preferences, and impairments. Once such a system has been set up, it can then be easily adapted to changing care needs and contexts of care.

\section{ACKNOWLEDGEMENTS}

We would like to thank Karl Isaac for assisting with the focus groups, and the people who invited us in to their 
homes for interviews, took part in our focus groups and filled in our survey. We would also like to thank Falkirk Sensory Services Centre, Hearing Concern Scotland, the Centre for the Older Person's Agenda, and Queen Margaret University for their support. This work was funded by EPSRC (EP/G060614/1, EP/G069387/1).

\section{REFERENCES}

1. Arking, R. Biology of Aging. Oxford University Press, New York, NY, $3^{\text {rd }}$ edition, 2005.

2. Biem, H. J., Turnell, R. W. and D'Arcy, C. Computer telephony: automated calls for medical care. Clin Invest Med, 26 (2003), 259-268.

3. Clark, J.S. and McGee-Lennon, M. R. A stakeholdercentred exploration of the current barriers to the uptake of home care technology in the UK. Journal of Assistive Technology, In Press (2011).

4. Consolvo, S., Roessler, P., Shelton, B. E., LaMarca, A., Schilit, B., and Bly, S. Technology for Care Networks of Elders. Pervasive Computing 3, 2 (2004), 22-29.

5. Department of Health. National Service Framework for Older People, London: The Stationery Office (2001).

6. Department of Health. Building Telecare in England. London: The Stationery Office (2005).

7. Deshpande, A. D., Harris-Hayes, M. and Schootman, M. Epidemiology of diabetes and diabetes-related complications. Phys Therapy, 88, 11 (2008), 12541264.

8. Garde, S. and Knaup, P. Requirements engineering in health care: the example of chemotherapy planning in pediatric oncology, Requirements Engineering 11 (2006), 265-278.

9. House of Lords Science and Technology Committee. Ageing: Scientific aspects. The Stationery Office, London, UK (2005).

10. Intille, S. S. A New Research Challenge: Persuasive Technology to Motivate Healthy Aging. IEEE Trans Inf Technol Biomedicine, 8, 3 (2004), 235-237.

11. Leonardi, C., Mennecozzi, C., Not, E., Pianesi, F., Zancanaro, M., Gennai, F. and Cristoforetti, A. Knocking on Elders' Door: Investigating the Functional and Emotional Geography of Their Domestic Space. In Proc. CHI 2009, ACM Press (2009), 1703-1712.

12. Ludford, P. J., Frankowski, D., Reily, K., Wilms, K., \& Terveen, L. Because I carry my cell phone anyway. In Proc. CHI 2006, ACM Press (2006), 889-898.

13. Mamykina, L., Miller, A. D., Mynatt, E. D., and Greenblatt, D. Constructing identities through storytelling in diabetes management. In Proc. CHI 2010 ACM Press (2010), 1203-1212.
14. McBryan, T. and Gray, P. A Model-Based Approach to Supporting Configuration in Ubiquitous Systems, In Proc. International Conference on Design, Specification and Verification of Interactive Systems, LNCS 5136, Springer (2008), 167-180.

15. McGee-Lennon, M.R. and Gray, P. Including Stakeholders in the Design of Home Care Systems: Identification and Categorisation of Complex User Requirements. In Proc. INCLUDE (2007).

16. McGee-Lennon, M.R., Wolters, M. and McBryan, T. Auditory Reminders in the Home. In Proc. ICAD 2007.

17. McGee-Lennon, M.R. Requirements engineering for home care technology. In Proc. CHI 2008, ACM Press (2008), 1439-1442.

18. McGee-Lennon, M.R., Wolters, M. K. and Brewster, S. Designing Reminders for the Home - The Role of Home Tours. Proc. INCLUDE (2011), In Press.

19. Morris, M., Lundell, J., Dishman, E., and Needham, B.. New Perspectives on Ubiquitous Computing from Ethnographic Study of Elders with Cognitive Decline Proc. UbiComp (2003), 227-242.

20. Mynatt, E. D., Melenhorst, A. S., Fisk, A. D., and Rogers, W. A. Aware technologies for aging in place: understanding user needs and attitudes. IEEE Pervasive Computing (2004), 3, 36-41.

21. Ofcom UK. Digital lifestyles - adults aged 60 and over, Report (2009).

22. Persad, U., Langdon, P. and Clarkson, J. Characterising user capabilities to support inclusive design evaluation. Universal Access in the Information Society, 6, 2 (2007), 119-153.

23. Pollack, M. Intelligent Technology for an Aging Population: The Use of AI to Assist Elders with Cognitive Impairment. AI Magazine 26 (2005), 9-24.

24. Ritchie, J. and Lewis, J. Qualitative Research Methods. Sage, London UK, 2003.

25. SENSE for deafblind people UK. A sense of urgency, Report, 2010.

26. Stanton, N., ed. Human factors in alarm design, Taylor and Francis, London UK, 1994.

27. S. Waller, P. Langdon, and P. Clarkson. Using disability data to estimate design exclusion. Univ Acc Inf Soc, 9 (2010), 195-207.

28. Watson, M. and Sanderson, P. Sonification Supports Eyes-Free Respiratory Monitoring and Task TimeSharing. Human Factors, 46, 3 (2004), 497-517

29. Wolters, M. K. and McGee-Lennon, M.R. Designing usable and acceptable reminders for the home. Proc. AAATE, 2010. 